\title{
Transcranial magnetic stimulation-evoked EEG/cortical potentials in physiological and pathological aging
}

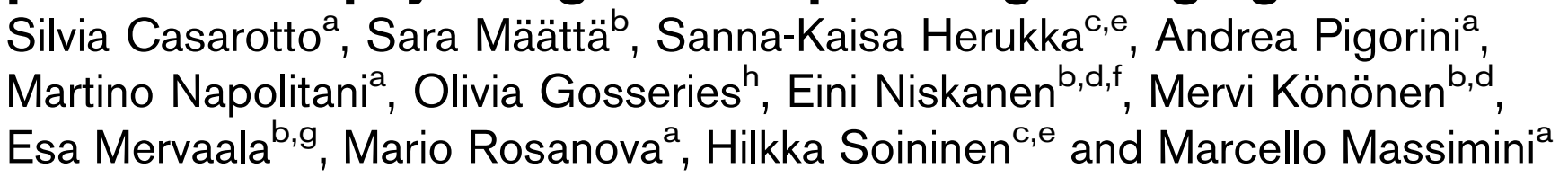

The frontal cortex undergoes macrostructural and microstructural changes across the lifespan. These changes can be entirely physiological, such as the ones occurring in elderly individuals who are cognitively intact, or pathological, such as the ones occurring in patients with Alzheimer's disease. Here, we use simultaneous electroencephalography (EEG) and transcranial magnetic stimulation (TMS) to study how the excitability of the frontal cortex changes during healthy and pathological aging. Hence, we compared the TMS-evoked EEG potentials collected in healthy elderly individuals with the ones collected in healthy young individuals, and in patients with Alzheimer's disease. We have shown that the EEG response to TMS of the left superior frontal cortex is not affected by physiological aging but is markedly altered by cognitive impairment. NeuroReport

\section{2:592-597 (c) 2011 Wolters Kluwer Health | Lippincott Williams \& Wilkins.}

NeuroReport 2011, 22:592-597

Keywords: aging, Alzheimer, cortical excitability, electroencephalography, transcranial magnetic stimulation

aDepartment of Clinical Sciences 'L. Sacco', Università degli Studi di Milano, Milan, Italy, ${ }^{\mathrm{b}}$ Departments of Clinical Neurophysiology, ${ }^{\mathrm{C}}$ Neurology, ${ }^{\mathrm{d}}$ Clinical Radiology, Kuopio University Hospital, ${ }^{e}$ Departments of Neurology, ${ }^{\mathrm{f}}$ Applied Physics, ${ }^{9}$ Clinical Neurophysiology, University of Eastern Finland, Kuopio, Finland and ${ }^{\mathrm{h}}$ Coma Science Group, Cyclotron Research Center, University of Liège, Liège, Belgium

Correspondence to Marcello Massimini, MD, PhD, Department of Clinical Sciences 'L. Sacco', Università degli Studi di Milano, Via G.B. Grassi 74, Milan 20157, Italy

Tel: + 3902 50319885; fax: + 390250319706 ;

e-mail: marcello.massimini@unimi.it

Received 15 April 2011 accepted 27 May 2011

together, suggest that TEPs may be used to detect and track over time pathological changes of cortical excitability.

As many neurological conditions that affect cortical function, such as cerebrovascular and degenerative diseases, tend to occur in the later stages of life, it would be important to know how TEPs change with aging. Aging is associated with an overall cerebral atrophy and with shrinkage of white and gray matter volumes, especially in the prefrontal cortex [13]. As shown by postmortem studies, these physiological alterations are more likely related to a loss of neuropil associated with a reduction of synapses and axons, rather than to a loss of neurons. As the electric field induced by TMS acts primarily on cortical axons and excites cortical neurons trans-synaptically [14], one may ask whether physiological aging, per se, may systematically affect the amplitude of TMS-evoked potentials.

In this study, we compared the immediate EEG responses to TMS of frontal cortex recorded in healthy elderly individuals with the ones collected in healthy young individuals, and found no significant differences. In contrast, we found markedly reduced TMS-evoked potentials in a population of elderly patients with Alzheimer's disease. By showing that TMS-evoked cortical potentials do not change with healthy aging, but change markedly with cognitive impairment, these experiments suggest that navigated TMS/EEG may be 
useful in detecting and tracking pathological changes in the state of cortical circuits across the lifespan.

\section{Methods \\ Participants}

This study involved nine healthy young individuals, nine healthy elderly individuals, and nine patients with Alzheimer's disease (Table 1). A neurological screening was performed to exclude potential adverse effects of TMS. Healthy individuals underwent clinical examinations to rule out a history or the presence of any relevant medical disorder. Patients with Alzheimer's disease were recruited from the local neurological outpatient clinic and were diagnosed according to the National Institute of Neurological and Communicative Disorders and StrokeAlzheimer's Disease and Related Disorders Association criteria for probable Alzheimer's disease [15]. All participants gave written informed consent to participate, and the study was approved by the Ethics Committee of Università degli Studi di Milano, by the Ethics Committee of Faculty of Medicine, University of Liege, and by the Ethics Committee of Kuopio University Hospital.

\section{Transcranial magnetic stimulation targeting}

TMS was delivered with a Focal Bipulse 8-Coil (for details see [12,16]) driven by a Mobile Stimulator Unit (Eximia TMS Stimulator; Nexstim Ltd., Helsinki, Finland) on the left superior frontal cortex (Brodmann's areas BA6/8) using structural magnetic resonance images (MRIs) acquired at $1.2-\mathrm{mm}^{3}$ spatial resolution (1.5T Siemens Magnetom Avanto; Erlangen, Germany). The actual location of the stimulating site (Fig. 1a) was individually adjusted to prevent the accidental activation of muscular fibers that inevitably affects EEG recordings. A Navigated Brain Stimulation (NBS) system (Nexstim Ltd) equipped with a three-dimensional infrared Tracking Position Sensor Unit (Polaris, Northern Digital Inc., Waterloo, Canada) was used to monitor the relative position between TMS coil and the patient's head. The integration of individual MRIs within this system provided a real-time estimation of the electric field induced on the cortical surface by TMS pulse and therefore allowed to precisely control (overall accuracy, $5.7 \mathrm{~mm}$ [17]) the actual stimulation parameters during each session. TMS intensity was set at approximately $110 \mathrm{~V} / \mathrm{m}$ on the cortical surface (Fig. 1b). Interstimulus interval was randomly jittered between $1.5-1.8 \mathrm{~s}$ (equivalent to about $0.5-0.6 \mathrm{~Hz}$ ). During TMS stimulation, patients wore inserted earplugs continuously playing a masking noise that abolishes the auditory potentials elicited by TMS-associated clicks [5].

\section{Electroencephalography recording}

A 60-channel TMS-compatible EEG amplifier (Nexstim Ltd) was used to record artifact-free neurophysiological responses to single TMS pulses, starting from $8 \mathrm{~ms}$ after stimulation [3]. Impedance at all electrodes was kept below $5 \mathrm{k} \Omega$. To monitor ocular movements and blinks, vertical electrooculogram was recorded with two extra electrodes. EEG signals were referenced to the forehead, were band-pass filtered between 0.1 and $500 \mathrm{~Hz}$, and were sampled at $1.450 \mathrm{~Hz}$ with 16 -bit resolution. The actual position of EEG electrodes on the scalp was digitized and coregistered with individual MRIs by the NBS system. EEG was continuously recorded during stimulation and was thereafter split into trials lasting $1.4 \mathrm{~s}$ and centered around TMS pulses. A minimum of 200 stimuli were delivered in each session. During the experiment, participants were lying on an ergonomic chair, relaxed, and with eyes open looking at a fixation point on a screen.

\section{Electroencephalography preprocessing}

Data analysis was carried out using Matlab (2006a, The MathWorks, Natick, Massachusetts, USA). Ocular artifacts were automatically reduced by applying principal component analysis. Thereafter, raw data were visually inspected by trained experimenters to reject from further analysis the single trials and channels residually contaminated by ocular and/or muscular artifacts. Artifactfree trials were band-pass filtered between $2-80 \mathrm{~Hz}$,

Table 1 Demographic characteristics (mean \pm standard deviation)

\begin{tabular}{|c|c|c|c|c|c|}
\hline \multirow[b]{2}{*}{ Group } & \multirow{2}{*}{$\begin{array}{l}\text { Healthy young } \\
\text { individuals }\end{array}$} & \multirow{2}{*}{$\begin{array}{c}\text { Healthy elderly } \\
\text { individuals }\end{array}$} & \multirow{2}{*}{$\begin{array}{c}\text { Patients with } \\
\text { Alzheimer's disease }\end{array}$} & \multicolumn{2}{|c|}{ Kruskal-Wallis ANOVA } \\
\hline & & & & $\chi_{2}^{2}$ & $P$ \\
\hline Individuals & 9 & 9 & 9 & - & - \\
\hline Age (years) & $31 \pm 4.5$ & $72 \pm 8.4^{\star *}$ & $72 \pm 7.1^{\star *}$ & 17.43 & 0.0005 \\
\hline Male/female & $7 / 2$ & $4 / 5$ & $4 / 5$ & - & - \\
\hline Education (years) & $18 \pm 3.0$ & $10 \pm 1.9^{*}$ & $10 \pm 3.5^{\star}$ & 16.08 & 0.0005 \\
\hline MMSE & $29 \pm 1.1^{\ddagger}$ & $28 \pm 1.3^{\ddagger}$ & $18 \pm 4.5$ & 17.89 & 0.0001 \\
\hline CDR total & $0 \pm 0.0^{\ddagger}$ & $0 \pm 0.0^{\ddagger}$ & $1 \pm 0.6$ & 24.88 & 0.000005 \\
\hline CDR sum of boxes & $0 \pm 0.0^{\ddagger}$ & $0 \pm 0.0^{\ddagger}$ & $7 \pm 3.6$ & 24.67 & 0.000005 \\
\hline GDS/FAST & $1 \pm 0.0^{\ddagger}$ & $1 \pm 0.0^{\ddagger}$ & $4 \pm 0.8$ & 24.81 & 0.000005 \\
\hline MT (\% stimulator maximum output) & $53 \pm 13.9$ & $50 \pm 10.4$ & $43 \pm 9.2$ & 4.30 & 0.12 \\
\hline MT (maximum induced electric field in V/m) & $88 \pm 13.3$ & $72 \pm 11.9$ & $75 \pm 18.8$ & 5.43 & 0.07 \\
\hline
\end{tabular}

CDR, Clinical Dementia Rating; GDS/FAST, Global Deterioration Scale/Functional Assessment Staging; MT, motor threshold; MMSE, Mini-Mental State Examination. Wilcoxon rank-sum test:

*Bonferroni-corrected $P<0.005$ compared with healthy young individuals.

**Bonferroni-corrected $P<0.0005$ compared with healthy young individuals.

${ }^{\ddagger}$ Bonferroni-corrected $P<0.0005$ compared with the patients with Alzheimer's disease. 
Fig. 1

(a)

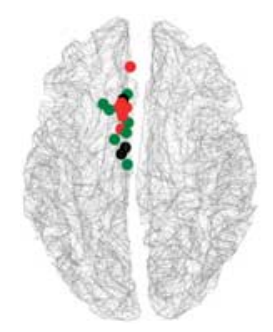
Location of individual TMS hotspots
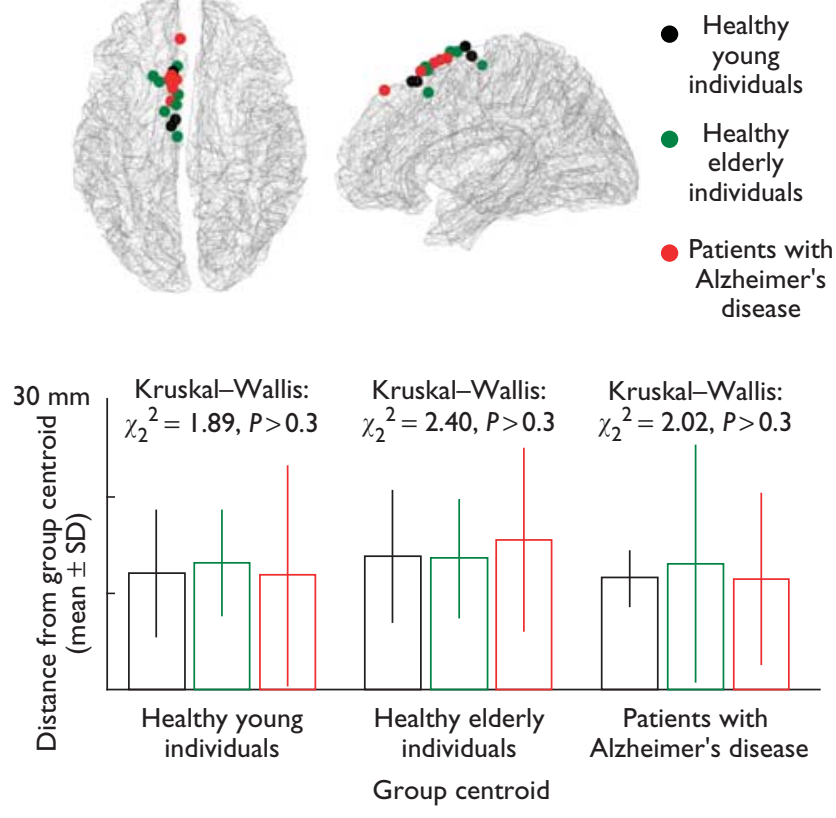

(b)

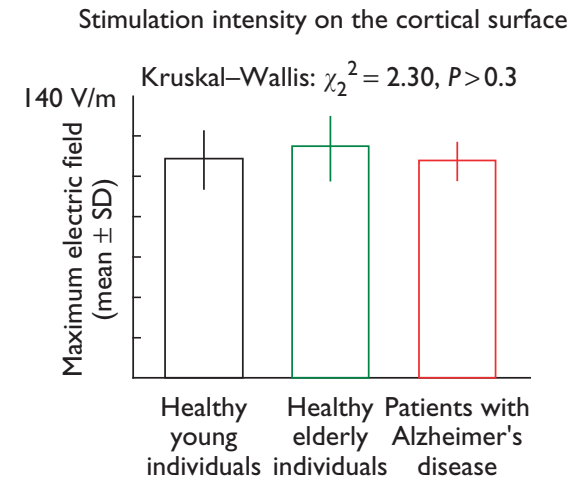

(a) Location of individual transcranial magnetic stimulation (TMS) hotspots (i.e. maximum induced electric field) on the cortical surface mesh (top). Distance between the individual TMS hotspots of each group (black bars for healthy young individuals, green bars for healthy elderly individuals, and red bars for patients with Alzheimer's disease) and the centroids of the three groups (bottom): Kruskal-Wallis analysis of variance showed that all centroids were equally distant from the TMS hotspots of each group. (b) Stimulation intensity (in V/m) on the cortical surface estimated by the navigation software in each group of participants; the maximum electric field induced by TMS pulses on the cortex was not significantly different among groups. SD, standard deviation.

downsampled to $725 \mathrm{~Hz}$, and rereferenced to the common average reference.

\section{Source modeling}

The three-spheres Berg method was adopted as a conductive head model (Brainstorm, http://neuroimage.usc. edu/brainstorm). Cortical surface was modeled as a threedimensional mesh of 3004 fixed dipoles by warping the Montreal Neurological Institute atlas to individual MRIs
(SPM5, http://www.fil.ion.bpmf.ac.uk/spm). EEG sensor positions and individual meshes were coregistered by rigid rotations and translations of anatomical landmarks (nasion, left and right tragus). Cortical sources were estimated from TEPs recorded on the scalp using the empirical Bayesian approach [16].

\section{Excitability measurement}

A nonparametric permutation-based statistical analysis was implemented to detect the spatiotemporal distribution of cortical sources significantly activated by TMS pulses [16]. Following Casali et al. [16], frontal cortex excitability was defined as the amplitude of the early and local neural response to TMS perturbation [1], and was measured by integrating the absolute current value of significantly activated sources (significant current density, SCD) over a temporal window immediately after the stimulation and over a cortical region surrounding the stimulated site. Time and space boundaries were empirically defined based on the real data (see Results section). Comparison among groups was made using nonparametric statistical analysis, namely Kruskal-Wallis analysis of variance and post-hoc Wilcoxon rank-sum test.

\section{Results}

Demographic characteristics of the population (Table 1) show that healthy elderly individuals and patients with Alzheimer's disease had comparable mean age and were older than healthy young individuals $(P<0.0005)$. Furthermore, the level of education (years) was higher in the healthy young group in comparison with healthy elderly and Alzheimer's disease groups $(P<0.0005)$. Patients with Alzheimer's disease were characterized by a considerable impairment of cognitive functions, as shown by lower scores on the Mini-Mental State Examination $(P<0.0001)$ and by higher scores on the Clinical Dementia Rating total, Clinical Dementia Rating sum of boxes, and Global Deterioration Scale/Functional Assessment Staging tests $(P<0.000005)$ compared with both healthy young and healthy elderly individuals. Finally, motor threshold, i.e. minimal TMS intensity to produce motor-evoked potentials of approximately $50-\mu \mathrm{V}$ amplitude with 0.5 probability in a stimulation sequence [18], was not significantly different among groups, both considering the percentage of the maximal stimulator output and the maximal electric field induced on the cortical surface estimated by the NBS system.

Homogeneity of stimulation parameters (in particular location and intensity) across participants is a basic requirement for group analysis of TEPs. To verify that location of the stimulated site did not differ among participants, the average spatial coordinates of individual TMS hotspots (centroids) were computed for each group separately. Then, Kruskal-Wallis analysis of variance was applied to compare the euclidean distances between the individual TMS hotspots of one group and the three 
group centroids. Results showed that each group was equally distant from all three centroids (Fig. 1a), meaning that the location of TMS hotspots was comparable among all participants $(P>0.3)$. The maximum electric field $(\mathrm{V} / \mathrm{m})$ induced on the frontal cortex automatically estimated by the NBS system was compared among all groups; we did not find group differences $(P>0.3)$, which means that all participants had been stimulated at the same intensity.

In this study, TEPs analysis was focused on the early and local response to TMS, a viable indicator of cortical excitability. The temporal window of interest (early) was set by looking at the grand average TEPs recorded in channel FC1 under the stimulator (Fig. 2a, right); the first relevant component elicited by TMS pulses occurred between 10 and $45 \mathrm{~ms}$. The cortical region surrounding the stimulated site (local) was defined, for each patient separately, as composed by the current sources located less than $3 \mathrm{~cm}$ (along the geodesic) from the individual TMS hotspot. This parameter is consistent with the size of the TMS stimulating area [1]; furthermore, as shown in Fig. 2b (left), it allowed to obtain an appropriate overlap among all participants (yellow) around the TMS target (i.e. left superior frontal cortex, BA6/8). At the group level, the temporal profile of SCD index averaged over the cortical region surrounding individual TMS hotspots (Fig. 2b, right) was characterized by two components between 10 and $45 \mathrm{~ms}$. These components were mainly evident in the grand average of healthy and elderly groups compared with the one of Alzheimer's disease group. The local mean SCD integrated over $10-45 \mathrm{~ms}$ (excitability index; Fig. 2c, left) turned out to be significantly different among groups $(P<0.01)$. Specifically, in patients with Alzheimer's disease, excitability was reduced compared with healthy young individuals (Bonferroni-corrected $P<0.05$ ) and healthy elderly individuals (Bonferronicorrected $P<0.01)$.

To verify whether this reduction was primarily due to the intensity of cortical currents or to the number of activated sources, a similar group comparison was applied to the percentage of sources significantly activated at any time sample in the cortical region surrounding TMS hotspots (Fig. 2c, middle) and to the SCD integrated over $10-45 \mathrm{~ms}$ and was averaged over the significant sources locally activated by TMS (Fig. 2c, right). Results showed that both the amount of current $(P<0.05)$ and the percentage of significant sources $(P<0.01)$ contribute to the reduced early and local response to TMS in patients with Alzheimer's disease compared with healthy young and elderly individuals. Indeed, SCD of the early response to TMS averaged over locally activated sources was lower in Alzheimer's disease compared with healthy young and elderly groups (Bonferroni-corrected $P<0.05)$; similarly, the percentage of locally activated sources was reduced (Bonferroni-corrected $P<0.05$ compared with healthy young individuals, and $P<0.01$ compared with healthy elderly individuals).

\section{Discussion}

This study demonstrates that TEPs are not affected by physiological aging per se, unless an abnormal cognitive decline (Alzheimer's disease) is associated. Thus, we found that frontal cortex excitability, that is, the early and local cortical response to TMS, was not significantly different between healthy young and elderly individuals, and was clearly reduced in elderly patients with Alzheimer's disease.

Healthy aging has been related to measurable changes in brain morphometry [13], including a decrease of wholebrain gray matter volume, surface area, and average thickness. This age-related atrophy is especially relevant in the bilateral prefrontal cortex. To date, this is the first study that investigates directly the changes of frontal cortex excitability as a function of healthy aging. Previous works have mainly focused on motor corticospinal excitability as measured by motor-evoked potentials [19]. Similar to our results, these studies also failed to find significant differences in corticospinal excitability between healthy young and elderly individuals.

Frontal lobes are known to be markedly involved in neurodegenerative diseases affecting cognitive functions, such as Alzheimer's disease. Although we found that TMSevoked potentials were not affected by normal aging, we detected a clear-cut impairment of frontal cortex excitability in patients with Alzheimer's disease compared with both young and elderly healthy individuals. This finding confirms and extends the results of a previous pilot study, in which the primary motor cortex was stimulated [20]; in this case, the comparison between patients with Alzheimer's disease and age-matched controls showed a reduction of global EEG response to TMS between 30 and $50 \mathrm{~ms}$, and a lower current density in the stimulated cortex at approximately $30 \mathrm{~ms}$ after TMS pulses. Previous studies, using TMS-evoked motor potentials (MEPs) have found a reduced motor threshold in patients with Alzheimer's disease compared with healthy controls, and have interpreted this result as an increased excitability of the corticospinal motor system [21-23]. These findings have been ascribed to an impairment of inhibitory GABAergic activity; alternatively, muscle tone, which has a facilitation effect on MEPs [24] and which was found to be increased in Alzheimer's disease [25], may play a role. Clearly, reconciling the increased corticospinal excitability as measured by MEPs with the reduced motor and frontal cortex excitability, as measured by TMS/EEG, is beyond the scope of this study.

\section{Conclusion}

This study demonstrates that, once the cortical electric field induced by TMS is standardized across subjects, TEPs 
Fig. 2

(a)

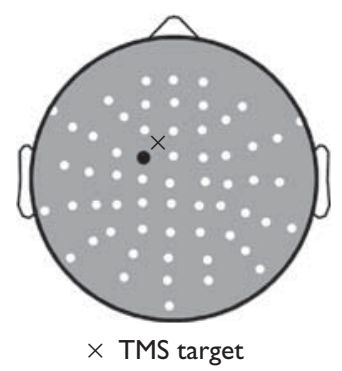

(b)

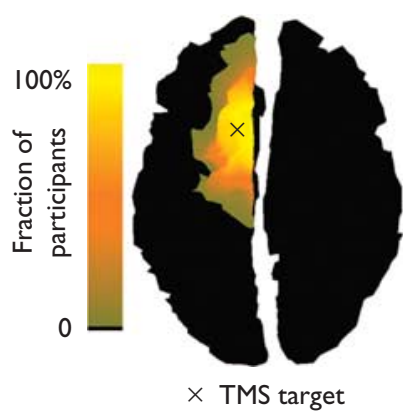

(c)
SCD of early response averaged across local sources

$\mathrm{KW}: \chi_{2}{ }^{2}=9.95, P<0.01$

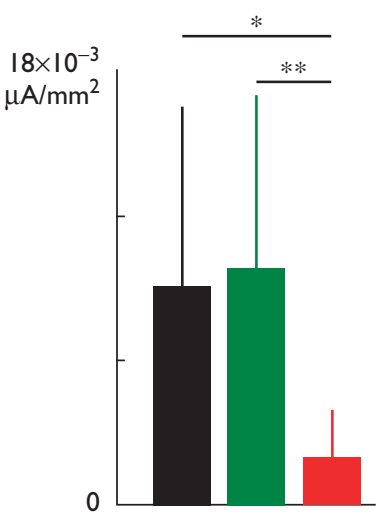

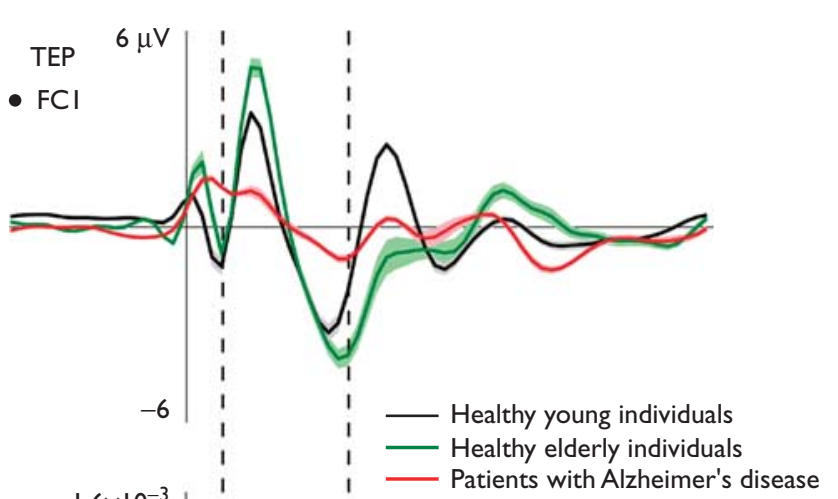

SCD

averaged over local sources

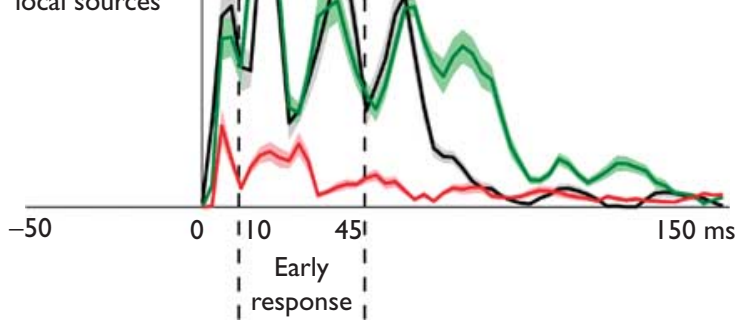

Percent of significant sources locally activated

$\mathrm{KW}: \chi_{2}^{2}=9.95, P<0.01$

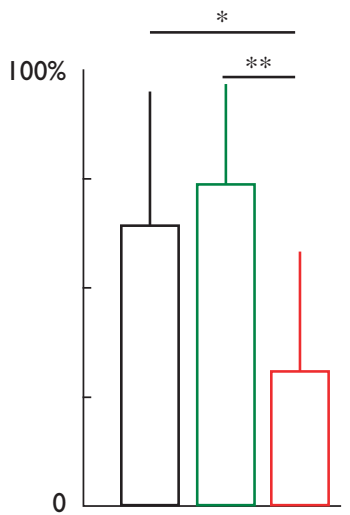

SCD of early response averaged across local significant sources

$\mathrm{KW}: \chi_{2}{ }^{2}=7.50, P<0.05$

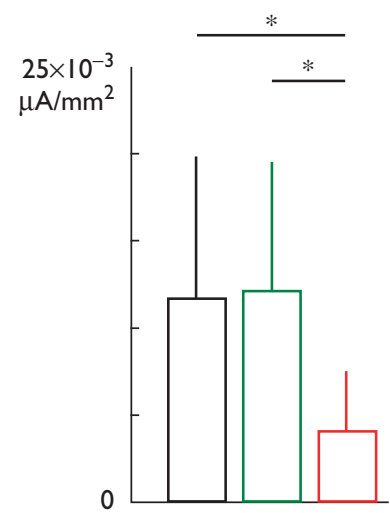

Healthy young Healthy elderly individuals individuals
Patients with Alzheimer's disease

(a) Spatial arrangement of electroencephalogram (EEG) electrodes on the scalp (left); transcranial magnetic stimulation (TMS) target and electrode FC1 located under the stimulator are highlighted. Grand average TMS-evoked potentials (TEPs) recorded from FC1 (right) in the three groups of participants. Colored shadows represent the instantaneous standard error across individuals in each group. The first component evoked by TMS occurred between 10 and $45 \mathrm{~ms}$ (dashed vertical lines). (b) Projection of the TMS target on the cortical surface (left); colored area contains the cortical sources located less than $3 \mathrm{~cm}$ (along the geodesic) from the individual TMS hotspot in any participant; the fraction of participants contributing to each source is color coded. Time course of the grand average SCD averaged over the cortical area under the stimulator (right). (c) Group comparison [Kruskal-Wallis analysis of variance (KW)] for the local mean SCD (left), for the percentage of sources significantly activated under the stimulator (middle), and for the SCD averaged across local significant sources (right) integrated over the early temporal window of interest of the cortical response to TMS [mean and standard deviation (SD)]. Bonferroni-corrected post-hoc Wilcoxon rank-sum test: ${ }^{\star} P<0.05,{ }^{\star \star} P<0.01$. 
are not significantly affected by aging, unless a pathological process, such as Alzheimer's disease, is involved. Stimulation parameters should always be carefully controlled for by means of a navigation system that maps the relative positions of TMS coil and patient's head within the reference space of an individual structural MRI and estimates the distribution and intensity of the intracranial electric field actually induced by TMS, using a realistic head model. We conclude that navigated TMS/EEG methodology may be effectively used to noninvasively detect and track pathological changes in the state of cortical circuits across the lifespan.

\section{Acknowledgements}

This work was supported by the European Grant Strep ICT-2007-224328 'Predict AD' (to M.M.).

The study was carried out at the Università degli Studi di Milano (data recording from healthy volunteers and data analysis), at the University of Liège (data recording from healthy volunteers), and at the Kuopio University Hospital (data recording from healthy volunteers and patients with Alzheimer's disease).

The authors thank Adenauer G. Casali, Matteo Fecchio, Simone Sarasso, and Paola Canali for their help and comments.

\section{References}

1 Ilmoniemi RJ, Virtanen J, Ruohonen J, Karhu J, Aronen HJ, Näätänen R, et al. Neuronal responses to magnetic stimulation reveal cortical reactivity and connectivity. Neuroreport 1997; 8:3537-3540.

2 Komssi S, Kähkönen S. The novelty value of the combined use of electroencephalography and transcranial magnetic stimulation for neuroscience research. Brain Res Rev 2006; 52:183-192.

3 Virtanen J, Ruohonen J, Näätänen R, Ilmoniemi RJ. Instrumentation for the measurement of electric brain responses to transcranial magnetic stimulation. Med Biol Eng Comput 1999; 37:322-326.

4 Ilmoniemi RJ, Kičić D. Methodology for combined TMS and EEG. Brain Topogr 2010; 22:233-248.

5 Massimini M, Ferrarelli F, Huber R, Esser SK, Singh H, Tononi G. Breakdown of cortical effective connectivity during sleep. Science 2005; 309:2228-2232.

6 Ferrarelli F, Massimini M, Peterson MJ, Riedner BA, Lazar M, Murphy MJ, et al. Reduced evoked gamma oscillations in the frontal cortex in schizophrenia patients: a TMS/EEG study. Am J Psychiatry 2008; 165:996-1005.

7 Thut G, Pascual LA. A review of combined TMS-EEG studies to characterize lasting effects of repetitive TMS and assess their usefulness in cognitive and clinical neuroscience. Brain Topogr 2010; 22:219-232.
8 Ferreri F, Pasqualetti P, Maatta S, Ponzo D, Ferrarelli F, Tononi G, et al. Human brain connectivity during single and paired pulse transcranial magnetic stimulation. Neuroimage 2011; 54:90-102.

9 Rosanova M, Casali A, Bellina V, Resta F, Mariotti M, Massimini M. Natural frequencies of human corticothalamic circuits. J Neurosci 2009; 29:7679-7685.

10 Kähkönen S, Wilenius J, Komssi S, Ilmoniemi RJ. Distinct differences in cortical reactivity of motor and prefrontal cortices to magnetic stimulation. Clin Neurophysiol 2004; 115:583-588.

11 Lioumis P, Kičić D, Savolainen P, Mäkelä JP, Kähkönen S. Reproducibility of TMS-evoked EEG responses. Hum Brain Mapp 2009; 30: 1387-1396.

12 Casarotto S, Romero Lauro L, Bellina V, Casali AG, Rosanova M, Pigorini A, et al. EEG responses to TMS are sensitive to changes in the perturbation parameters and repeatable over time. PLoS One 2010; 5:e10281.

13 Lemaitre H, Goldman AL, Sambataro F, Verchinski BA, Meyer-Lindenberg A, Weinberger DR, et al. Normal age-related brain morphometric changes: nonuniformity across cortical thickness, surface area and gray matter volume? Neurobiol Aging 2011 (in press).

14 Terao Y, Ugawa Y. Basic mechanisms of TMS. J Clin Neurophysiol 2002; 19:322-343.

15 McKhann G, Drachman D, Folstein M, Katzman R, Price D, Stadlan EM. Clinical diagnosis of Alzheimer's disease: report of the NINCDS-ADRDA Work Group under the auspices of Department of Health and Human Services Task Force on Alzheimer's Disease. Neurology 1984; 34:939-944.

16 Casali AG, Casarotto S, Rosanova M, Mariotti M, Massimini M. General indices to characterize the electrical response of the cerebral cortex to TMS. Neuroimage 2010; 49:1459-1468.

17 Ruohonen J, Ilmoniemi RJ. Modeling of the stimulating field generation in TMS. Electroencephalogr Clin Neurophysiol Supp/ 1999; 51:30-40.

18 Rossini PM, Barker AT, Berardelli A, Caramia MD, Caruso G, Cracco RQ, et al. Non-invasive electrical and magnetic stimulation of the brain, spinal cord and roots: basic principles and procedures for routine clinical application: report of an IFCN committee. Electroencephalogr Clin Neurophysiol 1994; 91:79-92.

19 Rossini PM, Rossi S, Babiloni C, Polich J. Clinical neurophysiology of aging brain: from normal aging to neurodegeneration. Prog Neurobio/ 2007; 83:375-400.

20 Julkunen $\mathrm{P}$, Jauhiainen AM, Westerén-Punnonen S, Pirinen E, Soininen $\mathrm{H}$ Könönen $\mathrm{M}$, et al. Navigated TMS combined with EEG in mild cognitive impairment and Alzheimer's disease: a pilot study. J Neurosci Methods 2008; 172:270-276.

21 Ferreri F, Pauri F, Pasqualetti P, Fini R, Dal Forno G, Rossini PM. Motor cortex excitability in Alzheimer's disease: a transcranial magnetic stimulation study. Ann Neurol 2003; 53:102-108.

22 Alagona G, Bella R, Ferri R, Carnemolla A, Pappalardo A, Costanzo E, et al. Transcranial magnetic stimulation in Alzheimer disease: motor cortex excitability and cognitive severity. Neurosci Lett 2001; 314:57-60.

23 Di Lazzaro V, Oliviero A, Tonali PA, Marra C, Daniele A, Profice P, et al. Noninvasive in vivo assessment of cholinergic cortical circuits in $A D$ using transcranial magnetic stimulation. Neurology 2002; 59:392-397.

24 Clark S, Tremblay F, Ste-Marie D. Differential modulation of corticospinal excitability during observation, mental imagery and imitation of hand actions. Neuropsychologia 2004; 42:105-112.

25 Kischka U, Mandir AS, Ghika J, Growdon JH. Electrophysiologic detection of extrapyramidal motor signs in Alzheimer's disease. Neurology 1993; 43:500-505. 